UDK 528.21

\title{
GEOID PRECISION FROM LIMITED-AREA GRAVIMETRIC SURVEYS
}

\author{
Martin Vermeer ${ }^{1}$, Karin Kollo ${ }^{2}$ \\ ${ }^{1}$ Helsinki University of Technology (TKK), Espoo, Finland \\ E-mail: martin.vermeer@tkk.fi \\ ${ }^{2}$ Estonian Land Board, Tallinn, Estonia \\ E-mail: karin.kollo@maaamet.ee
}

Received 1107 2006, accepted 21122006

\begin{abstract}
We derive a number of rough theoretical estimates for the precision of a geoid model computed from a local gravimetric survey combined with global reference model information. Example calculations for Finland and Estonia are presented.
\end{abstract}

Keywords: geoid precision, gravimetric survey.

\section{Introduction}

It is possible to give a number of theoretical estimates for the precision of a gravimetric geoid computed using gravimetric survey data obtained from a bounded area and having a finite density of measurement points. The derivations presented below have in the past appeared in different form and in small pieces in the nonreviewed literature, often in connection with practical geoid determination projects [1-3]. These derivations, which are of simple, brute-force nature, are summarized and presented more clearly and systematically in the present article, while we also take the opportunity to correct some errors present in the earlier derivations.

We will consider three main types of geoid error deriving from a gravity survey's limited nature:

- The error of omission due to the finite spatial density of the gravity survey.

- The aliasing error, also due to the finite spatial density of the survey, where error patterns with halfwavelengths shorter than this spacing are misrepresented as geoid error patterns with longer wavelengths.

- The out-of-area error, caused by the absence of gravity survey coverage outside the area of study. Gravity information may be missing completely; or it may be available as long wavelength only information, eg as a spherical harmonic expansion of a reference gravity model. Estimating the out-of-area error requires knowledge of the signal covariance function, either of the full gravity field, or of the part of gravity not described by the reference model.

As input to the expressions we derive we need mainly two quantities:

- The mean separation of gravimetric measurement points, and
- the average „error of prediction“ in the form of a mean error for an arbitrary point somewhere in the terrain, when predicting its gravity anomaly from those of nearby points. This error is in principle computable if we know two quantities defining the gravity anomaly field's signal covariance function: its signal covariance $C_{0}$ and its correlation length $l$.

For the Finnish gravimetric survey, values have been quoted [2, eg] of $d=5 \mathrm{~km}$ and $\sigma_{\Delta g}= \pm 2 \mathrm{mGal}$. These values probably hearken back to a determination made already in 1980 [4] but is undoubtedly still valid today.

For Estonia in recent years, two geoid model computations have been undertaken: [5] and [6]. For the gravimetric survey used in this work we have the values $d=4 \mathrm{~km}$ and $\sigma_{\Delta g}= \pm 3 \mathrm{mGal}$. The value of $3 \mathrm{mGal}$ originates from Artu Ellmann's licentiate thesis [5]; in his Doctoral dissertation he gives [7] a value of 2,4 mGal. However, one of us (KK) after discussing with Tõnis Oja, concluded that the value $3 \mathrm{mGal}$ is probably more realistic.

\section{The error of omission}

If we have gravimetric data given on a grid with spacing $d$, the Nyquist theorem tells us that the grid points can only represent a low-pass filtered anomaly field with cut-off wavelength $2 d$, ie degree $n=2 \pi R / 2 d$.

If the mean error of prediction of gravity $\sigma_{\Delta g}$ is given, which we may assume equal to the RMS of the degree variances above the truncation limit; and if we assume for simplicity that all power is concentrated at $n=\pi R / d$, then we can compute (using, as in the sequel, $\left.\gamma=9,81 \mathrm{~m} \mathrm{~s}^{-2}\right)$ : 


$$
\sigma_{N}=\frac{\sigma_{\Delta g} R}{(n-1) \gamma}=\frac{d \sigma_{\Delta g}}{\pi \gamma}=0,3 d \sigma_{\Delta g}
$$

if we express $d$ in $\mathrm{km}, \sigma_{\Delta g}$ in $\mathrm{mGal}$ and $\sigma_{N}$ in $\mathrm{mm}$. This near-trivial derivation appeared first in [1]. Obviously this is only an upper bound; gravity anomaly power will in reality be present in all degrees between $n=\pi R / d$ and $\infty$.

For the Finnish gravimetric survey and values quoted above, one obtains for the error of omission in the geoid undulation: $\sigma_{N}= \pm 3 \mathrm{~mm}$. For Estonia, using the values quoted above, we similarly obtain $\sigma_{N}=3,6 \mathrm{~mm}$.

\section{The aliasing error (1)}

If the true gravity anomaly field we are trying to represent is not low-pass filtered in this way, we will have an aliasing error caused by the part of the field above the truncation degree.

If we assume for a moment the grid to be regular with spacing $d$, and the high-frequency part of the $\Delta g$ field to be uncorrelated at different grid point locations, with mean error $\sigma_{\Delta g}$, then this is white noise. If the grid is of size $D \times D$ (ie point counts $\frac{D}{d}, \frac{D}{d}$ in $x$ and $y$ directions), then there will be $\left(\frac{2 D}{d}\right)^{2}$ spectral components in both co-ordinate directions, with wavelengths $D, \frac{D}{2}, \frac{D}{3}, \ldots, d$, with each both a real and an imaginary part. Or equivalently, wavelengths running from $-d, \ldots,-\frac{D}{2},-D, \infty,+D, \frac{D}{2}, \ldots, d$.

For white noise, each of these constituents will then have amplitude $\sigma_{\Delta g} \frac{d}{2 D}$ in order to produce the given total RMS power.

Computing the corresponding geoid error means summing up the squares of these contributions, which are

$$
\sigma_{\Delta g} \frac{d}{2 D} \frac{D}{\sqrt{i^{2}+j^{2}}} \frac{1}{\pi \gamma}
$$

where $i$ and $j$ both run from $-\frac{D}{2}$ to $+\frac{D}{2}$ but with $i=j=0$ excluded, something to keep in the backs of our minds throughout the below derivation.

We may approximate this sum by an integral:

$$
\begin{aligned}
& \sum_{i=-D / d}^{D / d} \sum_{j=-D / d}^{D / d} \frac{D^{2}}{i^{2}+j^{2}}= \\
& D^{2} \int_{-1 / d}^{+1 / d} \int_{-1 / d}^{+1 / d} \frac{1}{u^{2}+v^{2}} d u d v
\end{aligned}
$$

over the rectangle $\left[-\frac{1}{d}, \frac{1}{d}\right] \times\left[-\frac{1}{d}, \frac{1}{d}\right]$. Here, $u=\frac{i}{D}$ and $v=\frac{j}{D}$ are wave numbers in the two co-ordinate directions. This again is approximated by the integral over a circular disc with a hole in the middle, with $p=\sqrt{u^{2}+v^{2}}$ :

$$
\begin{aligned}
& D^{2} \int_{-1 / d}^{+1 / d} \int_{-1 / d}^{+1 / d} \frac{1}{u^{2}+v^{2}} d u d v= \\
& D^{2} \int_{0}^{2 \pi} \int_{1 / D}^{1 / d} \frac{1}{p^{2}} p d p d \alpha= \\
& 2 \pi D^{2} \int_{1 / D}^{1 / d} \frac{1}{p} d p=2 \pi D^{2} \ln \left(\frac{D}{d}\right),
\end{aligned}
$$

because now we integrate over $p$ from $\frac{1}{D}$ (remember, the origin $u=v=0$, ie the geographic $(x, y)$ area outside the $D \times D$ square, is excluded!) to $\frac{1}{d}$ (more precise bounds would be from $\frac{4}{\pi D}$ to slightly less than $\frac{1}{d}$ ).

The total result then is

$$
\begin{aligned}
& \sigma_{N}=\sigma_{\Delta g} \frac{d}{2 D} \sqrt{2 \pi D^{2} \ln \left(\frac{D}{d}\right)} \frac{1}{\pi \gamma}= \\
& \sigma_{\Delta g} \frac{d}{2} \sqrt{2 \pi \ln \left(\frac{D}{d}\right)} \frac{1}{\pi \gamma}= \\
& \frac{d}{\sqrt{2 \pi}} \frac{\sigma_{\Delta g}}{\gamma} \sqrt{\ln \frac{D}{d}} .
\end{aligned}
$$

Compared to the expression (1) found earlier, this will typically be dominant. Note that whereas $d$ is the spacing of the $\Delta g$ data points, $D$ represents the scale at which geoid errors are constrained, eg by GPS/levelling points, or by a global geopotential reference model.

\subsection{Application to the Finnish gravimetric survey}

Cf Table 1, recomputed from an earlier version appearing in [2]. The same values for $d$ and $\sigma_{\Delta g}$ were used as earlier.

Table 1. The aliasing error in Finland as a function of geoid support point separation $D$

\begin{tabular}{|c|c|c|c|}
\hline$D(\mathrm{~km})$ & $\sigma_{N}(\mathrm{~mm})$ & $D(\mathrm{~km})$ & $\sigma_{N}(\mathrm{~mm})$ \\
\hline 10 & 3,4 & 100 & 7,0 \\
\hline 20 & 4,8 & 200 & 7,8 \\
\hline 50 & 6,2 & 500 & 8,7 \\
\hline
\end{tabular}




\subsection{Application to the Estonian gravimetric survey}

Cf Table 2, computed using the values referred to earlier.

Table 2. The aliasing error in Estonia as a function of geoid support point separation $D$

\begin{tabular}{|c|c|c|c|}
\hline$D(\mathrm{~km})$ & $\sigma_{N}(\mathrm{~mm})$ & $D(\mathrm{~km})$ & $\sigma_{N}(\mathrm{~mm})$ \\
\hline 10 & 4,7 & 100 & 8,8 \\
\hline 20 & 6,2 & 200 & 9,7 \\
\hline 50 & 7,8 & 500 & 10,7 \\
\hline
\end{tabular}

\section{The aliasing error (2)}

An independent derivation starts from the Stokes integral. In the near-field limit the Stokes kernel is $\frac{2}{\psi}=\frac{2}{R r}$, with $r$ the geometric distance. Now consider an area $D \times D$ in size, within which there are $\left(\frac{D}{d}\right)^{2}$ gravity anomaly point values given with mean error $\sigma_{\Delta g}$, each of which is representative of a small square of size $d \times d$. Again, we must compute the sum of square contributions from all these squarelets. Each such contribution is

$$
\left(\frac{R}{4 \pi \gamma}\right)^{2}\left(\frac{2 d^{2}}{R r}\right)^{2}=\frac{d^{4}}{r^{2}}\left(\frac{1}{2 \pi \gamma}\right)^{2}
$$

We must sum this (the expression on the left in $d$ and $r$ ) over all little squares of size $d \times d$ in the big square of size $D \times D$. Alternatively, integrate:

$$
\iint \frac{d^{2}}{r^{2}} d x d y
$$

where $x, y$ are plane metric coordinates. This integral is from $-\frac{D}{2}$ to $+\frac{D}{2}$ in both coordinates.

Again go to polar coordinates with $r=\sqrt{x^{2}+y^{2}}$, yielding:

$$
2 \pi d^{2} \int \frac{1}{r} d r
$$

between limits $\frac{d}{2}$ [we exempt a small disc around the origin where the integral would misbehave] and $\frac{D}{2}$.

This yields

$$
2 \pi d^{2} \ln \left(\frac{D / 2}{d / 2}\right)=2 \pi d^{2} \ln \frac{D}{d} .
$$

Back substitution yields

$$
\sigma_{N}=\sigma_{\Delta g} d \sqrt{2 \pi \ln \frac{D}{d}} \frac{1}{2 \pi \gamma}=\frac{d}{\sqrt{2 \pi}} \frac{\sigma_{\Delta g}}{\gamma} \sqrt{\ln \frac{D}{d}}
$$

identical to Eq (2).

\section{The out-of-area error}

The two above calculations assume an infinite extent of the gravimetric survey data. In reality this data will always be limited in extent, and then error will be generated especially in the border areas due to the lack of gravimetric data on the other side of the border.

We assume that the lacking gravimetric data - or alternatively, the lacking short-wavelength part of the gravimetry not contained in a global reference model used - can be described by the parameters of a Hirvonen signal covariance function

$$
C(r)=\frac{C_{0}}{1+r^{2} / l^{2}}
$$

where the two defining parameters are the signal variance $C_{0}$ and the correlation length $l . r$ is the inter-point distance.

In order to determine the error, we apply a discretised Stokes integration. We divide the area to the other side of the border into squares of size $l$, ie we choose $\Delta x=\Delta y=l$, the correlation length:

$$
N=\frac{l^{2}}{2 \pi \gamma} \sum_{i, j} \frac{\Delta g_{i j}}{r_{i j}}
$$

Here, $i$ and $j$ are block subscripts in the two geographical directions $x$ and $y$, and the ,blocks“ of gravimetric data are of size $l \times l$.

For simplicity, we consider a straight line border only, extending to infinity. On the left side of the border, good gravimetric data is available. On the right side, we have a missing component in the gravimetric data which is described by $C_{0}$ and $l$. We assume the $l \times l$ size blocks to be statistically independent from each other, with each being characterised by a signal variance of $C_{0}$. Eq (5):

Then we obtain using propagation of variances on

$$
\begin{aligned}
& \sigma_{N}^{2}=\left(\frac{l^{2}}{2 \pi \gamma}\right)^{2} \sum_{i, j} C_{0} \frac{\Delta x \Delta y}{l^{2}} r_{i j}^{-2}= \\
& \frac{C_{0} l^{2}}{4 \pi^{2} \gamma^{2}} \sum_{i, j} r_{i j}^{-2} \Delta x \Delta y .
\end{aligned}
$$


Now we replace the right-hand side sum by an integral:

$$
\begin{aligned}
& \sum_{i, j} r_{i j}^{-2} \Delta x \Delta y=\int_{-\infty}^{+\infty} \int_{\delta}^{\infty} \frac{1}{x^{2}+y^{2}} d x d y= \\
& \int_{\delta}^{\infty}\left[\int_{-\infty}^{+\infty} \frac{1}{x^{2}+y^{2}} d y\right] d x= \\
& \int_{\delta}^{\infty} \frac{1}{x^{2}}\left[\int_{-\infty}^{+\infty} \frac{1}{1+y^{2} / x^{2}} d y\right] d x= \\
& \int_{\delta}^{\infty} \frac{1}{x}\left[\int_{-\infty}^{+\infty} \frac{1}{1+w^{2}} d w\right] d x= \\
& \int_{\delta}^{\infty} \frac{1}{x}[\arctan (\infty)-\arctan (-\infty)] d x= \\
& \pi \int_{\delta}^{\infty} \frac{1}{x} d x=\pi \ln \frac{\infty}{\delta},
\end{aligned}
$$

where $\delta$ is the distance of the evaluation point from the border. We have here used a plane coordinate system centered upon the evaluation point $x=0, y=0$, while the border is $y=\delta$. See the figure.

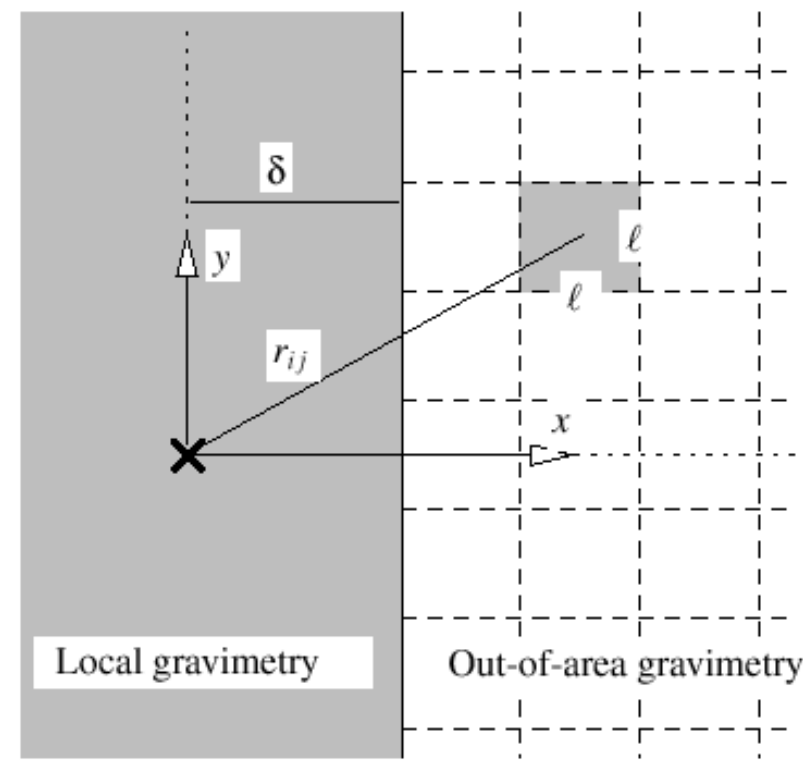

Geometry for estimating out-of-area geoid error

Clearly the plane approximation breaks down here we get an infinite total error. Also for the limit $\delta \rightarrow 0$ the result diverges. Both these effects are non-physical. We eliminate the lower bound problem by requiring it to be at least $l$, ie we replace $\delta$ by $\max (\delta, l)$.

For the upper bound, we introduce a limiting value $\lambda$ equal to the semi-wavelength for which the global reference model becomes precise. For current models that would be $n_{\max }=20, \quad$ corresponding to $\lambda=20,000 \mathrm{~km} / 40=1000 \mathrm{~km}$; once the GOCE results are in, we would more likely have $n_{\max }=100$ or $\lambda=200 \mathrm{~km}$. Then our integral becomes

$$
\int_{-\sqrt{\lambda^{2}-x^{2}}}^{+\sqrt{\lambda^{2}-x^{2}}} \int_{\max (\delta, l)}^{\lambda} \frac{1}{x^{2}+y^{2}} d x d y
$$

integrating over a disc of radius $\lambda$ around the evaluation point. Instead of this difficult integral, we use the upper bound

$$
\begin{aligned}
& \int_{-\infty}^{+\infty} \int_{\max (\delta, l)}^{\lambda} \frac{1}{x^{2}+y^{2}} d x d y= \\
& \int_{\max (\delta, l)}^{\lambda}\left[\int_{-\infty}^{+\infty} \frac{1}{x^{2}+y^{2}} d y\right] d x= \\
& \int_{\max (\delta, l)}^{\lambda} \frac{1}{x} d x=\pi \ln \frac{\lambda}{\max (\delta, l)}
\end{aligned}
$$

like above. Obviously, also for $\lambda<\delta$ this would become negative $(\lambda<l$ would be unphysical), so we change this to

$$
\pi \ln \frac{\max (\delta, \lambda)}{\max (\delta, l)} .
$$

These are obviously very crude tricks.

So now we have

$$
\sigma_{N}^{2}=\frac{C_{0} l^{2}}{4 \pi \gamma^{2}} \ln \frac{\max (\lambda, \delta)}{\max (l, \delta)}
$$

From Eq (4) we can derive the variance of the difference between two gravity anomalies in points $P$ and $Q$ as a function of point separation $d$ :

$$
\begin{aligned}
& \operatorname{Var}\left(\Delta g_{P}-\Delta g_{Q}\right)=\operatorname{Var}\left(\Delta g_{P}\right)+\operatorname{Var}\left(\Delta g_{Q}\right)- \\
& 2 \operatorname{Cov}\left(\Delta g_{P}, \Delta g_{Q}\right)=2 \frac{r_{P Q}^{2}}{l^{2}} \frac{C_{0}}{1+r_{P Q}^{2} / l^{2}} .
\end{aligned}
$$

This expression describes the prediction accuracy obtained when trying to predict the gravity anomaly $\Delta g_{Q}$ from the given value $\Delta g_{P}$, with the distance between points $P$ and $Q$ being $r_{P Q}$. Substituting $r_{P Q} \rightarrow d$, the average point spacing of the gravimetric survey, we may call this the variance of prediction, $\sigma_{\Delta g}^{2}$, of the survey:

$$
\sigma_{\Delta g}^{2}=2 \frac{C_{0}}{1+d^{2} / l^{2}} \frac{d^{2}}{l^{2}} .
$$

Inversion gives us 


$$
C_{0}=\frac{1}{2} \sigma_{\Delta g}^{2}\left(1+\frac{d^{2}}{l^{2}}\right) \frac{l^{2}}{d^{2}}=\frac{1}{2} \sigma_{\Delta g}^{2}\left(1+\frac{l^{2}}{d^{2}}\right)
$$

which we substitute into Eq (6), yielding

$$
\sigma_{N}^{2}=\frac{l^{2}}{8 \pi \gamma^{2}}\left(1+\frac{l^{2}}{d^{2}}\right) \sigma_{\Delta g}^{2} \ln \frac{\max (\lambda, \delta)}{\max (l, \delta)}
$$

Substituting the constants (like $\gamma=9,81 \mathrm{~m} \mathrm{~s}^{-2}$ ) and introducing units yields

$$
\begin{aligned}
& \sigma_{N}^{2}\left[\mathrm{~mm}^{2}\right]=4,1 \cdot 10^{-2} l^{2}\left[\mathrm{~km}^{2}\right]\left(1+\frac{l^{2}}{d^{2}}\right) \times \\
& \sigma_{\Delta g}^{2}\left[\mathrm{mGal}^{2}\right] \ln \frac{\max (\lambda, \delta)}{\max (l, \delta)}
\end{aligned}
$$

\subsection{Application to the Finnish gravimetric survey}

With this formula we have computed Table 3 for the Finnish gravimetric survey parameters $d$ and $\sigma_{\Delta g}$. A realistic value for the correlation length may well be $l=20 \mathrm{~km}$ or a little longer. As seen, improvement of the out-of-area gravity field brought about by the satellite gravity missions, bringing down the shortest wellrepresented half-wavelength from 500 to $100 \mathrm{~km}$, will somewhat improve the quality of the local geoid, though not spectacularly so.

Remember, though, that the values tabulated here only apply close to the border: the error will diminish going in-land (increasing $\delta$ ), though not very quickly, as $\delta$ appears within the logarithm. For $\delta \geq \lambda$ (and thus $\delta>l$ ), the logarithm will vanish. This will actually happen inland in Finland when the new GOCE geopotential model is available with its small $\lambda=200 \mathrm{~km}$. Let us remember that the values in Table 3 are valid only in the border zone, and the width of this border zone may be taken as the half-wavelength $\lambda$ of the global reference model used. Using a better (higherdegree) expansion will narrow down the border zone influenced by these errors, an additional benefit of GOCE that is not obvious from the Table: in the second rightmost column, the italicised value 27,80 represents the error $100 \mathrm{~km}$ inland from the border.

Thus, while a clear improvement is to be expected from these missions, it will not certainly do away with the need to obtain a good, dense gravimetric coverage for the problem areas immediately outside Finland's borders. Where terrestrial gravimetry is not available, airborne gravimetry recommends itself.

\subsection{Application to the Estonian gravimetric survey}

Doing the same computation for Estonia, with the earlier quoted parameter values $d$ and $\sigma_{\Delta g}$, yields the results listed in Table 4. Here one may guess that the correlation length in Estonia is a bit shorter than in Finland, more like $10 \mathrm{~km}$, producing plausible-looking geoid mean errors for a wide range of $(\lambda, \delta)$ combinations.

Otherwise the conclusions are similar to those for Finland. Due to the smallness of Estonia, the values in this table underestimate the true uncertainty of the absolute geoid, which will improve substantially due to the satellite missions. But also here, even more so, good gravimetric survey data immediately across the borders must also be obtained.

Table 3. Out-of-area gravimetric geoid standard deviation, Finnish gravimetric survey parameters: $\sigma_{\Delta g}= \pm 2 \mathrm{mGal}, d=5 \mathrm{~km}$ Units: $\mathrm{mm}$

\begin{tabular}{|c|c|c|c|c|c|c|c|}
\hline $\begin{array}{l}l \downarrow \max (\lambda, \delta) \rightarrow \\
\max (l, \delta) \rightarrow\end{array}$ & $\begin{array}{r}1000 \\
20\end{array}$ & $\begin{array}{r}200 \\
20\end{array}$ & $\begin{array}{r}1000 \\
50\end{array}$ & $\begin{array}{r}200 \\
50\end{array}$ & $\begin{array}{r}1000 \\
100\end{array}$ & $\begin{array}{l}200 \\
100\end{array}$ & $\begin{array}{l}200 \\
200\end{array}$ \\
\hline 10 & 32,49 & 24,92 & 28,43 & 19,34 & 24,92 & 13,67 & 0,00 \\
\hline 20 & 123,00 & 94,40 & 107,70 & 73,24 & 94,40 & 51,79 & 0,00 \\
\hline 50 & 756,50 & 580,40 & 662,00 & 450,30 & 580,40 & 318,40 & 0,00 \\
\hline 100 & 3019,00 & 2316,00 & 2642,00 & 1797,00 & 2316,00 & 1271,00 & 0,00 \\
\hline
\end{tabular}

\begin{tabular}{|l|r|r|r|r|r|r|r|}
\hline$l \downarrow \max (\lambda, \delta) \rightarrow$ & 1000 & 200 & 1000 & 200 & 1000 & 200 & 200 \\
$\max (l, \delta) \rightarrow$ & $\mathbf{2 0}$ & 20 & 50 & 50 & 100 & 100 & 200 \\
\hline 10 & 17,91 & 13,74 & 15,67 & 10,66 & 13,74 & 7,54 & 0,00 \\
\hline $\mathbf{2 0}$ & $\mathbf{6 6 , 0 5}$ & 50,67 & $\mathbf{5 7 , 8 0}$ & 39,32 & $\mathbf{5 0 , 6 7}$ & 27,80 & 0,00 \\
\hline 50 & 402,49 & 308,79 & 352,21 & 239,60 & 308,79 & 169,42 & 0,00 \\
\hline 100 & 1604,00 & 1230,60 & 1403,60 & 954,82 & 1230,60 & 675,16 & 0,00 \\
\hline
\end{tabular}

Table 4. Out-of-area gravimetric geoid standard deviation, Estonian gravimetric survey parameters: $\sigma_{\Delta g}= \pm 3 \mathrm{mGal}, d=4 \mathrm{~km}$ Units: mm 


\section{References}

1. VERMEER, M.; OLLIKAINEN, M. GPS and height determination. Geodesia, Vol 29, Otaniemi, 1995, p. 14-26.

2. VERMEER, M. Two new geoids determined at the FGI. Reports of the Finnish Geodetic Institute, 95:5, Masala: Finnish Geodetic Institute, 1995. 25 p.

3. VERMEER, M. The new Baltic Sea and Finnish geoids of the FGI. In Vilks, I. (editor). Studies of the Baltic Sea. Proceedings, First Workshop of the Subcommission IAG SSC 8.1, Riga, Latvia, March 28-29, 1996, p. 59-66.

4. KOSTIAINEN, M.; KAKKURI, J. On the accuracy of the gravity determined from the Bouguer anomaly register for leveling bench marks. In Proc Second International Symposium on Problems Related to the Redefinition of North American Geodetic Networks (NAD Symposium). Ottawa, Canada, May 26-30, 1980, p. 525-536.

5. ELLMANN, A. Least squares modification of Stokes formula with application to the Estonian geoid. Licentiate thesis. Stockholm: Royal Institute of Technology, 2001. $98 \mathrm{p}$.

6. JÜRGENSON, H. Determination of Estonian Precision Geoid. Doctoral dissertation. Tartu: Estonian Agricultural University, 2003. 157 p.

7. ELLMANN, A. An improved gravity anomaly grid and a geoid model for Estonia. In Proc Estonian Acad Sci, Geology, 51(4), 2002, p. 199-214.
Martin VERMEER, Prof, PhD, Helsinki University of Technology TKK, P. O. Box 1200, FI-02015 Espoo, Finland. $\mathrm{Ph}+35894513910$,

e-mail: martin.vermeer@tkk.fi.

Author of more than 80 scientific papers, 15 peer reviewed.

Research interests: geoid, GPS, co-ordinate systems and geodynamics, geodetic software and numerics.

Karin KOLLO, Chief specialist, Estonian Land Board, Mustamäe tee 51, Box 1635, 10602 Tallinn, Estonia, $\mathrm{Ph}+3726650619$, Fax +3726650604,

e-mail: karin.kollo@maaamet.ee.

Post-graduate student in Helsinki University of Technology, Dept of Surveying, Laboratory of Geoinformation and Positioning Technology.

Research interests: geoid, satellite gravimetry. 